\title{
CLASSIFICATION OF THE COMPLETELY PRIMARY TOTALLY RAMIFIED ORDERS WITH A FINITE NUMBER OF NONISOMORPHIC INDECOMPOSABLE LATTICES
}

BY K. W. ROGGENKAMP

Communicated by Hyman Bass, November 15, 1971

Let $K$ be the p-adic completion of an algebraic number field and denote by $R$ its ring of integers. We assume that $\Lambda$ is an $R$-order in the semisimple finite dimensional $K$-algebra $A$. One of the main problems in the representation theory of orders is the classification of those orders $\Lambda$ which have only a finite number of nonisomorphic indecomposable left $\Lambda$-lattices

the so-called "orders of finite lattice type." The commutative case has been settled independently by Drozd-Roiter [2] and Jacobinski [3]. For the general case only partial results are known [1], [4], [5], [7].

By Morita equivalence we may assume that $\Lambda / J(\Lambda)$, where $J(\Lambda)$ denotes the Jacobson-radical of $\Lambda$, is a finite direct sum of extension fields $\boldsymbol{R}_{i}$ of $\Re$, the residue field of $R$, say

$$
\Lambda / J(\Lambda) \stackrel{\text { ring }}{\cong} \bigoplus_{i=1}^{m} \Omega_{i}
$$

We choose a finite unramified extension $K^{\prime}$ of $K$ with ring of integers $R^{\prime}$ such that the residue field $\boldsymbol{R}^{\prime}$ of $R^{\prime}$ is a splitting field for the minimum polynomial of $\boldsymbol{\Omega}_{i}$ over $\boldsymbol{R}$. Putting $\Lambda^{\prime}=R^{\prime} \otimes_{R} \Lambda$ we have

$$
\Lambda^{\prime} / J\left(\Lambda^{\prime}\right) \cong \bigoplus_{i=1}^{n} \mathfrak{R}^{\prime}
$$

Jacobinski [3, Proposition 1] has shown that $\Lambda$ is of finite lattice type if and only if $\Lambda^{\prime}$ is of finite lattice type. Therefore we may assume that

$$
\Lambda / J(\Lambda) \cong \bigoplus_{i=1}^{n} \Re
$$

where $\boldsymbol{R}$ is the residue field of $R$.

We shall classify here those orders of finite lattice type for which $n=1$ - these are called "completely primary totally ramified" (notation CPTR). By $n(\Lambda)$ we denote the number of nonisomorphic indecomposable left $\Lambda$-lattices.

AMS 1970 subject classifications. Primary 16A18, 16A48, 16A64.

Key words and phrases. Orders, finite lattice type, completely primary orders.

Copyright $($ American Mathematical Society 1972 
THEOREM. Let $\Lambda$ be a CPTR-order in $A . n(\Lambda)<\infty$ if and only if either

(i) For every CPTR-overorder $\Omega$ of $\Lambda$ we have

$(\alpha)$ The left ring of multipliers $\Omega^{\prime}$ of $J(\Omega)$ coincides with the right ring of multipliers of $J(\Lambda)$.

Put $\mathfrak{U}=\Omega^{\prime} / J(\Omega)$,

$(\beta) \operatorname{dim}_{\mathfrak{A}}(\mathfrak{H}) \leqq 3$,

$(\gamma) \operatorname{dim}_{\mathscr{A}}\left(J(\mathfrak{H}) / J^{2}(\mathfrak{U})\right) \leqq 1$, where $J(\mathfrak{U})$ denotes the Jacobson-radical of $\mathfrak{A}$,

or

(ii) $\Lambda$ is conjugate to

$$
\Omega_{1}=\left\{\left(\begin{array}{ccc}
a_{11} & a_{12} & a_{13} \\
\pi^{\prime} a_{21} & a_{11}+\pi^{\prime} a_{22} & a_{12}+\pi^{\prime} a_{23} \\
\pi^{\prime} a_{31} & \pi^{\prime} a_{32} & a_{11}+\pi^{\prime} a_{33}
\end{array}\right) \mid a_{i j} \in R^{\prime}, 1 \leqq i, j \leqq 3\right\},
$$

where $R^{\prime}$ is a totally ramified finite extension of $R$ and $\pi^{\prime} R^{\prime}=J\left(R^{\prime}\right)$.

REMARK. $\Omega_{1}$ is the only type of CPTR-order with a finite number of nonisomorphic indecomposable lattices for which the left ring of multipliers of the radical is different from the right ring of multipliers of the radical.

SOME COMMENTS TO THE PROOF. Let

$$
A=\bigoplus_{i=1}^{s}\left(D_{i}\right)_{s_{i}},
$$

where $D_{i}$ are skewfields over $K$, and assume that $\Lambda$ is a CPTR-order in $A$ with $n(\Lambda)<\infty$. Then it is shown in [6] and [7] that

$$
\sum_{i=1}^{s} s_{i} \leqq 3
$$

One shows quite easily that for $s_{i}=1,1 \leqq i \leqq s$, the conditions of our theorem coincide with the conditions of Drozd-Roiter [2] (cf. also [5], [7]).

In case $s=1$ and $s_{1}=3$ one shows that $n\left(\Omega_{1}\right)<\infty$ using some results of Kirichenko [4], and with [7] this case is settled. For $s=1$ and $s_{1}=2$, one shows that the conditions of our theorem imply that $\Lambda$ is a Bass-order, and with [1], this case is settled. Finally for $s=2, s_{1}=1$ and $s_{2}=2$, one has to do some computations (similar to those in [2] and [5]) to conclude that our conditions are sufficient for $n(\Lambda)<\infty$.

REMARK. Let $\Lambda$ be any order satisfying (1). Let $1=\sum e_{i}$ be the decomposition of $1 \in \Lambda$ into primitive orthogonal idempotents. Then $\Omega_{i}=e_{i} \Lambda e_{i}$ is a CPTR-order and $n(\Lambda)<\infty$ implies $n\left(\Omega_{i}\right)<\infty$ and so $\Omega_{i}$ is known. 


\section{REFERENCES}

1. Ju. A. Drozd and V. V. Kiricenko, On representations of rings lying in matrix algebras of the second kind, Ukrain. Mat. Ż. 19 (1967), no. 3, 107-112. (Russian) MR 35 \#1632.

2. Ju. A. Drozd and A. V. Roìter, Commutative rings with a finite number of indecomposable integral representations, Izv. Akad. Nauk SSSR Ser. Mat. 31 (1967), 783-798 = Math. USSR Izv. 1 (1967), 757-772. MR 36 \# 3768.

3. H. Jacobinski, Sur les ordres commutatifs avec un nombre fini de réseaux indécomposables, Acta Math. 118 (1967), 1-31. MR 35 \# 2876.

4. V. V. Kiricenko, Representations of matrix rings of third order, Mat. Zametki 8 (1970), 235-244. (Russian)

5. K. W. Roggenkamp, Charakterisierung von Ordnungen in einer direkten Summe kompletter Schiefkörper, die nur endlich viele nicht isomorphe unzerfällbare Darstellungen haben, Mitt. Math. Sem. Giessen 89 (1971), 1-122.

6. - Some orders of infinite lattice type, Bull. Amer. Math. Soc. 77 (1971), 1055-1056.

7. ___ Some necessary conditions for orders to be of finite lattice type. I, II, J. Reine Angew. Math. (to appear).

Department of Mathematics, University of Bielefeld, Post FaCh 8640(48) Bielefeld, GERMANY 